This item was submitted to Loughborough's Research Repository by the author.

Items in Figshare are protected by copyright, with all rights reserved, unless otherwise indicated.

\title{
An intelligent and confident system for automatic surface defect quantification in 3D
}

PLEASE CITE THE PUBLISHED VERSION

http://www.ttp.net/978-3-03835-488-8/toc.html

PUBLISHER

(C) Trans Tech Publications Inc

VERSION

AM (Accepted Manuscript)

\section{PUBLISHER STATEMENT}

This work is made available according to the conditions of the Creative Commons Attribution-NonCommercialNoDerivatives 4.0 International (CC BY-NC-ND 4.0) licence. Full details of this licence are available at: https://creativecommons.org/licenses/by-nc-nd/4.0/

\section{LICENCE}

CC BY-NC-ND 4.0

\section{REPOSITORY RECORD}

Tailor, Mitul, Jon N. Petzing, and Michael R. Jackson. 2019. "An Intelligent and Confident System for Automatic Surface Defect Quantification in 3D”. figshare. https://hdl.handle.net/2134/18298. 


\title{
An intelligent and confident system for automatic surface defect quantification in 3D
}

\author{
Mitul Tailor, Jon Petzing, Michael Jackson \\ EPSRC Centre for Innovative Manufacturing in Intelligent Automation \\ Loughborough University, Loughborough, UK \\ M.Tailor@lboro.ac.uk
}

Keywords: Defect artefacts, Optical Measurement, Feature Quantification

\begin{abstract}
:
Automatic surface defect inspection within mass production of high-precision components is growing in demand and requires better measurement and automated analysis systems. Many manufacturing industries may reject manufactured parts that exhibit even minor defects, because a defect might result in an operational failure at a later stage. Defect quantification (depth, area and volume) is a key element in quality assurance in order to determine the pass or failure criterion of manufactured parts. Existing human visual analysis of surface defects is qualitative and subjective to varying interpretation. Non-contact and three dimensional (3D) analyses should provide a robust and systematic quantitative approach for defect analysis. Various 3D measuring instruments generate point cloud data as an output, although they work on different physical principles. Instrument's native software processing of point cloud data is often subject to issues of repeatability and may be non-traceable causing significant concern with data confidence.

This work reports the development of novel traceable surface defect artefacts produced using the Rockwell hardness test equipment on flat metal plate, and the development of a novel, traceable, repeatable, mathematical solution for automatic defect detection and quantification in 3D. Moreover, in order to build-up the confidence in automatic defect analysis system and generated data, mathematical simulated defect artefacts (soft-artefact) have been created. This is then extended to a surface defect on a piston crown that is measured and quantified using a parallel optical coherence tomography instrument integrated with 6 axis robot. The results show that surface defect quantification using implemented solution is efficient, robust and more repeatable than current alternative approaches.
\end{abstract}

\section{Introduction}

In industry, surface topography may be one of the significant factors in performance of high precision components. Surface topography is normally recognized as comprising of different surface components, i.e. roughness, waviness, form, and surface defects. Whilst separation of roughness, waviness and form components is usually conducted by the mean-line based filters [1], discrete detection of surface defects is also crucial because they may play very important roles in functional performance. Inspection of surface defects is a crucial task for aerospace industries, in terms of product quality, production efficiency, and performance efficiency. Any suspicious region requires assessment in terms of a pass / fail criterion.

Automatic detection of process-induced defects (e.g. indentations and scratches), is an important issue in machine vision. Detection of surface defects in 2D and 3D has been reported for various applications in different industries. Examples include; the real-time detection of defects on fruits and vegetables [2], the robust and automated detection of defects on transparent and non-plane surface defect [3], along with a computer-aided visual inspection system for surface defect detection in ceramic capacitor chips [4]. Once defects have been identified, it is important to accurately extract the defect from the surface. Several algorithms have been developed and published for defect detection in images [5-7] as well as different filtration techniques set out in the ISO 16610 series of standards [8] 
to aid the characterization of surface features [1][9]. This can also be adopted to detect surface defects. Once a defect is detected and ideally isolated, it then becomes important to quantify the defect geometry (such as depth, area and volume). Although significant work has been reported in detecting surface defects using different methods, robust and automatic methods for quantification of defect in $3 \mathrm{D}$, is less well explored.

ISO 8785 [10] gives the definition of types of surface defects but currently standards are not available to quantify defects. Currently ISO 25178-2 [11] is available to quantify aspects of surface volume of materials, which can and has been adopted to quantify surface defects. Commercial analysis software are also available that allow a user to delimit a defect manually and consequently calculate the geometric quantities. However visually driven manual delimiting of a defect is always subjective and qualitative, leading to repeatability/reproducibility issues and measurement errors. Hard-artefacts (comparators) and soft-artefacts are commercially available for surface texture analysis and validation of the software respectively. Such artefacts boost the confidence level in measurement process and provide traceable solution. However, there is an issue of reliability of data representation when it comes to defect measurement due to the lack of traceable surface defect artefact and soft-artefact.

In this research, repeatable and traceable defect artefacts have been generated and a novel algorithm has been developed and verified using created novel soft-artefact to quantify defects automatically in 3D.

\section{Defect artefacts}

The lack of commercial standard surface defect comparators hampers the work of standard surface defect artefacts generation. Artefacts have been created using repeatable Rockwell hardness test equipment which is typically used to determine the hardness of substrates. In this test, indentations are made using a known size conical indenter on a metal surface. Irrespective of the material hardness, these indentations have a unique shape and known geometry that can be used as defect artefacts to develop a robust defect quantification method.

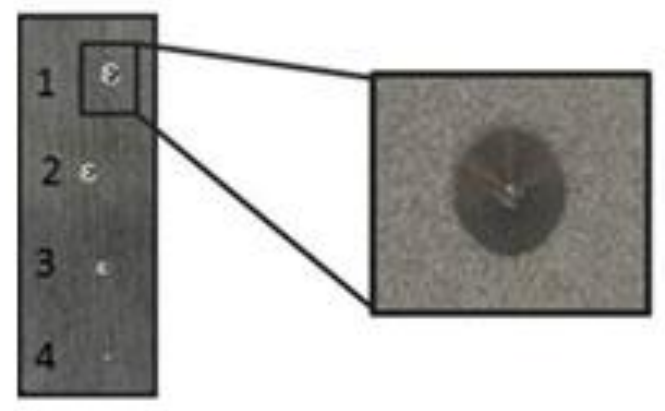

Figure 1 Defect artefacts

Four different sizes of conical impression have been generated on flat standard stainless steel following the specification set out in ISO 6508-1 [12]. These are nominally $300 \mu \mathrm{m}, 250 \mu \mathrm{m}, 180 \mu \mathrm{m}$ and $40 \mu \mathrm{m}$ in depth. Fig. 1 shows the four different sizes of Rockwell indentations on a flat plate.

Optical measurement of artefacts: The defect artefacts have been measured in three dimension using Heliotis H4 instrument. The Heliotis H4 is an optical instrument, typically works on the physical principle of parallel optical coherence tomography. The $3 \mathrm{D}$ optical sensor is capable of measuring different surface types, including ground and polished surfaces, steps and films. The instrument produces point cloud data with a field of view of approximately $2.4 \mathrm{~mm} \times 2.4 \mathrm{~mm}$ with a lateral resolution of approximately $8.0 \mu \mathrm{m}$. The measuring instrument is mounted on 6-axis Fanuc industrial robot-Mate 200ic. 


\section{Algorithm}

The novel algorithm to quantify the defect automatically has been created using MATLAB $\mathrm{R} 2012 \mathrm{~b}$ and the flow chart is briefly illustrated in Fig. 2. The optical measuring instrument provides point cloud data as the output of the measured surface. Fig. 3 illustrates point cloud data of the measured surface that contains conical defect 1 (Fig. 1).

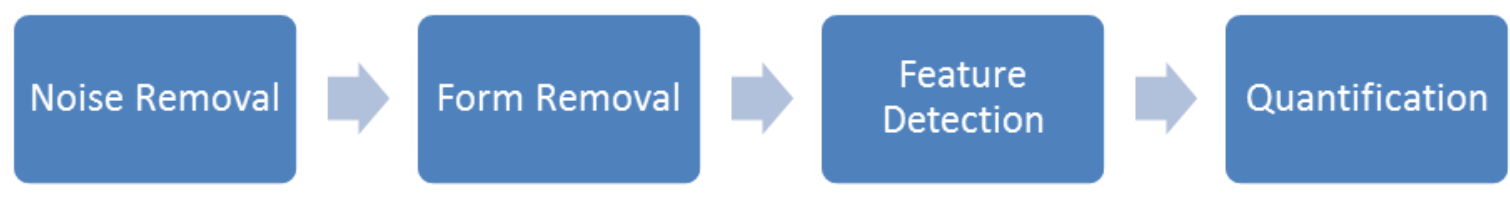

Figure 2 Defect quantification algorithm

Measurement noise which is high frequency component, for instance spurious spikes, is always present in measured data from any 3D optical instruments and it is necessary to eliminate such noise for better quantitative assessment otherwise it may lead to incorrect quantification. A low pass areal Gaussian filter is implemented to remove high frequency components. Moreover, 3D datasets also contain geometric form which is typically in the millimetric scale that needs to be removed for better assessment of the defect. If this process is not achieved, large scale form would mask smaller scale defect information. By generating the mean surface using robust Gaussian regression filter, form can be removed thus a residual surface can be obtained. After the filtration process, it is important to isolate the defect from the residual surface. The purpose of this process is to locate the defect region and 3D data portions for later defect quantification. For defect isolation, edge detection of the defect is essential. An edge of the defect is defined as an abrupt change in surface height on the 3D data. In this algorithm, local thresholding considering the surface texture element has been adopted to isolate the defect.

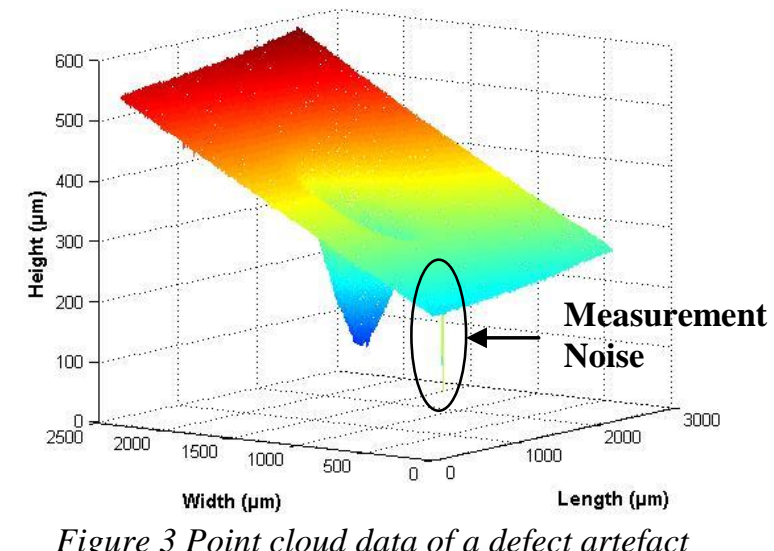

Once a defect is isolated, it is relatively straight forward to locate the boundary of the defect in 3D that can be clearly seen in Fig. 4 as a blue circumferential line outlining the brim of the defect region. A reference plane is generated using the least square method to fit into the defect boundary data points. In a given field of view, the algorithm tries to find the minimum pixel value. Once the minimum pixel value is obtained then perpendicular distance from the minimum point to the generated least square plane can be easily calculated using simple mathematical equation which is effectively depth information of the defect. To calculate a defect's area, total number of pixels encapsulated in the defect boundary region is considered. Once total number of pixels is known that covers defect area, then simple multiplication with lateral resolution would provide the area of a defect. To compute a volume of a defect, the algorithm calculates perpendicular distance from each defect points (pixel) within defect region to the least square plane. Considering an area of a pixel that is derived from a 
lateral resolution, the sum of all perpendicular distance from each pixel to the reference plane is ultimately the required volume of the defect.

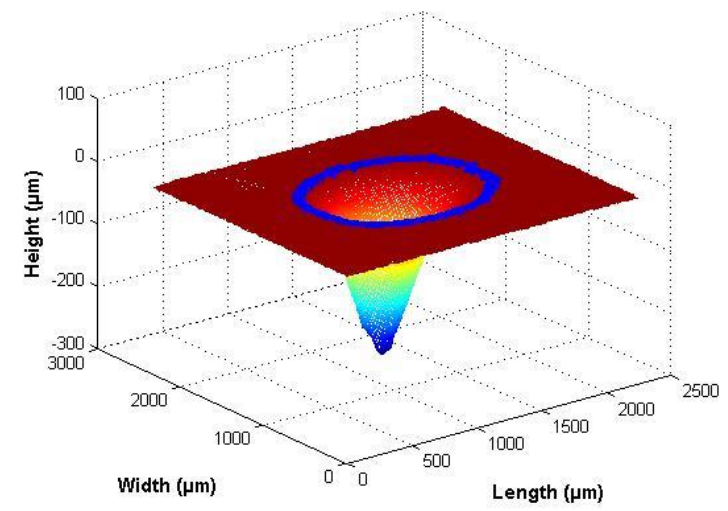

Figure 4 Isolated defect in residual surface

Generation of soft-artefact: It is important to validate the algorithm in order to verify the results and build-up confidence in the automatic defect analysis system and generated data. A novel defect soft-artefact is created using MATLAB R2012b in order to verify the implemented algorithm to quantify defect automatically in 3D. A mathematical geometric shaped defect of known size embedded in a theoretically flat plate can be used as a soft-artefact. The soft-artefact can potentially be implemented using two different methods. Firstly, theoretically well-defined soft-artefact can be designed and implemented using CAD design software. Secondly, the soft-artefact can be developed using an iterative process. However through investigation, it was observed that CAD software does not produce datasets in the form of point cloud data that is generated using typical 3D optical instruments. Thus an iterative process has been chosen to design and create a soft-artefact.

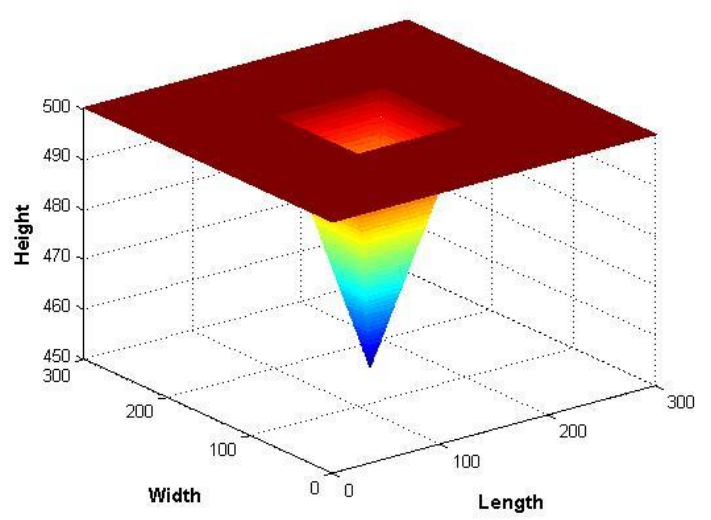

Figure 5 Pyramidal shape soft-artefact

Pyramidal shape soft-artefact has been created using iterative process (Fig. 5). Here it is noted that the soft-artefact is a mathematical model and hence each pixel represents just a number that does not have attributed dimension. Moreover the $\mathrm{x}$ axis, $\mathrm{y}$ axis and $\mathrm{z}$ axis represent length, width and height respectively. For the particular soft-artefact, the length, width and height of the soft-artefact are 100 units, 100 units and 50 units respectively. The depth of the soft-artefact is equal to the absolute height of the soft-artefact. As the softgauge has a known geometry, area and volume can be easily calculated using standard mathematical equation. Thus theoretical values of depth, area and volume for the soft-artefact are 50 units, 10000 units and 166666.67 units respectively. 
Validation of algorithm: In order to validate the novel algorithm, the soft-artefact with the specification mentioned in above section was measured using the algorithm. Measurement results from the algorithm should match the theoretical values in order to validate the algorithm that leads to traceability to the mathematically defined size geometry. The soft-artefact was processed and the novel algorithm isolated the defect from the surface as shown in Fig. 6 as a blue circumferential line outlining the throat of the pyramidal defect region.

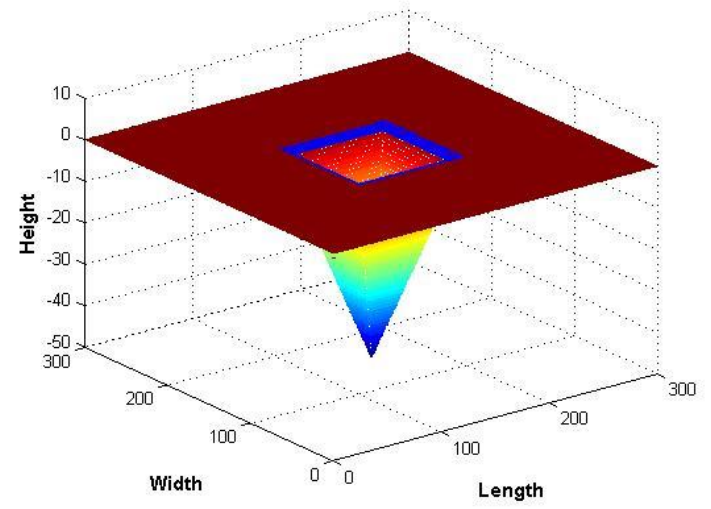

Figure 6 Isolated soft-artefact

With a resolution of 1 unit, the depth, area and volume of the defect were computed as 49 unit, 9801 unit and 156820 unit respectively. The novel algorithm measured the depth of the defect with $2.0 \%$ of measurement error, the area of the defect region with $1.99 \%$ measurement error and the volume of the defect with $5.91 \%$ measurement error. Here measurement error is a function of resolution. By increasing the resolution (effectively decreasing the pixel size), the measurement error can be minimized. To demonstrate this phenomenon, soft-artefact has been generated with resolution of 0.1 unit. It is observed that the algorithm computes depth of 49.9 unit with $0.2 \%$ measurement error, area of 9980 unit with $0.2 \%$ measurement error and volume of 165670 unit with $0.6 \%$ measurement error. Hence, by improving the resolution, the measured geometrical quantities of the soft-artefact approach the theoretical values. Hence, the algorithm to quantify surface defects is validated using a developed novel soft-artefact.

\section{Results}

Automatic defect quantification of the different sizes of defect artefacts using the algorithm is shown in Table 1. In this example, artefacts shown in Fig. 1 are measured five times repetitively using Heliotis H4. It can be seen that the relative standard deviation in calculating geometrical quantities (depth, area and volume) is observed to be less than $0.4 \%$ in defects above $177.0 \mu \mathrm{m}$ in depth. However, the algorithm computes depth, area and volume of the smallest defect (38.0 $\mu \mathrm{m}$ in depth) with slightly higher relative standard deviation than rest of the other defect which is approximately $1.0 \%-3.0 \%$. The higher variation is observed in measuring smallest defect due to uncertainty associated with measuring instrument and generation of defect artefacts. In the context of this work, relative standard deviation is used for better representation of experimental data and for effective graphical representation of the data. Logarithmic scales are used due to the high magnitude of the quantities. Fig. 7 is a graphical representation of the geometric parameters of the four different size defects on a logarithmic scale 
Table 1Quantification of defect artefacts

\begin{tabular}{|c|c|c|c|c|c|c|}
\hline \multirow{2}{*}{$\begin{array}{c}\text { Defect } \\
\text { ID }\end{array}$} & \multicolumn{3}{|c|}{ Average Measured Quantities } & \multicolumn{3}{c|}{ \% Standard Deviation } \\
\cline { 2 - 7 } & Depth $(\mu \mathrm{m})$ & Area $\left(\mu \mathrm{m}^{2}\right)$ & Volume $\left(\mu \mathrm{m}^{3}\right)$ & Depth & Area & Volume \\
\hline 1 & 304 & 1426074 & 87888000 & 0.1 & 0.3 & 0.1 \\
\hline 2 & 252 & 1281856 & 57007400 & 0.1 & 0.3 & 0.2 \\
\hline 3 & 177 & 1135027 & 24831500 & 0.2 & 0.2 & 0.3 \\
\hline 4 & 38 & 28603 & 431164 & 1.2 & 1.5 & 2.7 \\
\hline
\end{tabular}

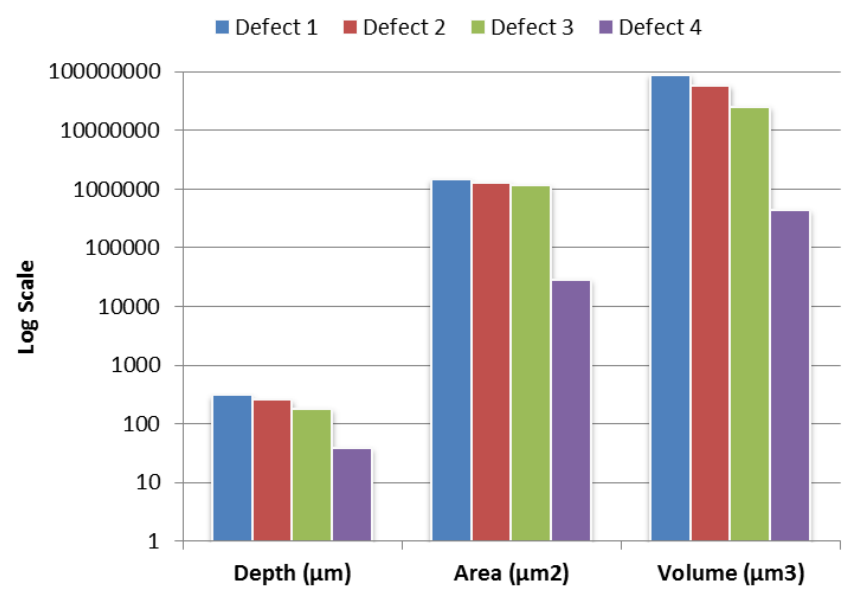

Figure 7 Comparison of measured quantities of artefact of different sizes

\section{Industrial application}

Automotive industries may reject parts with defects in the manufacturing process, because even a minor defect in a manufactured part may cause a functional failure at a later stage. Thus it is very important to detect defects at an early stage and (as in this case) to quantify depth, area and volume of the suspicious region because these are often key parameters in quality assurance in order to check the health of the manufactured component.

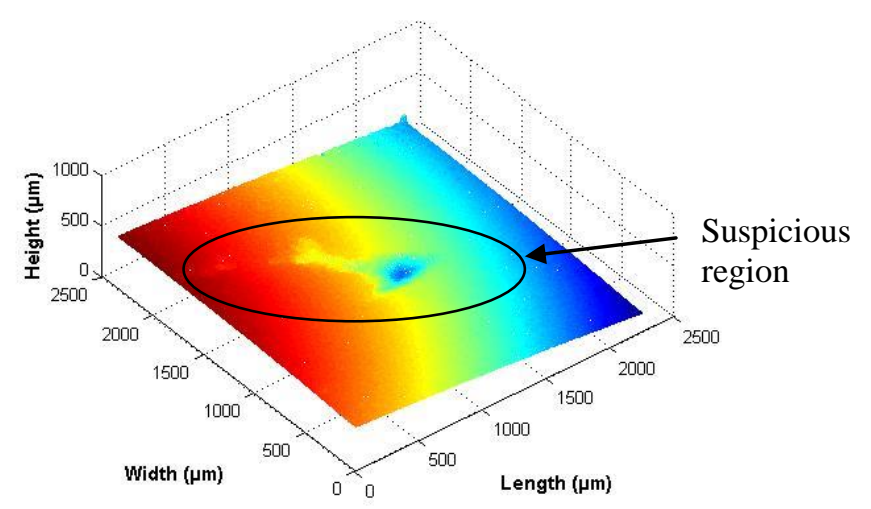

Figure 8 Raw data of the piston crown suspicious region

Fig. 8 shows the 3D data of suspicious region of a piston crown. It can be seen that the region is masked by the large scale of geometric form, with the suspicious region highlighted in Fig. 8. It thus follows that the precise detection of the suspicious region, isolation from the surrounding substrate, and its quantification, is very critical. 


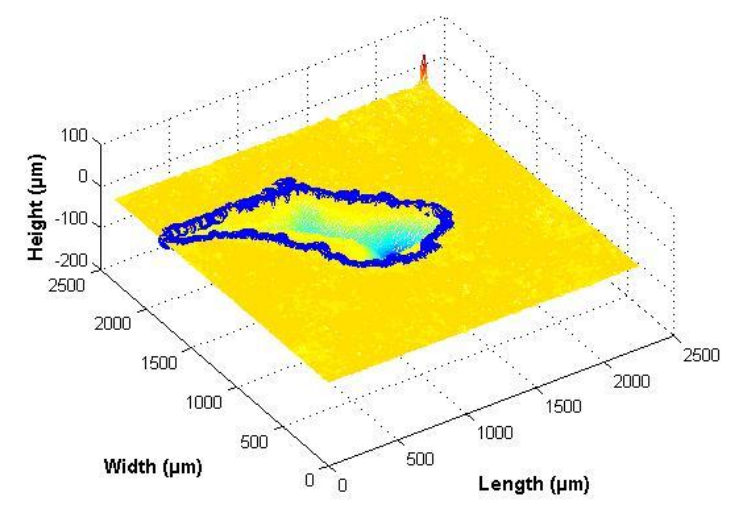

Figure 9 Isolated suspicious region in residual surface

Measured 3D data of the suspicious region on the piston crown is processed using the implemented and verified algorithm. Fig. 9 illustrates the form-free residual surface with the highlighted suspicious region boundary as a blue circumferential line. The novel algorithm computes the maximum depth as being $115 \mu \mathrm{m}$, area as being $973752 \mu \mathrm{m}^{2}$ and measures the volume as being $11682200 \mu^{3}$ with an approximate relative standard deviation of $1.4 \%$ in all.

\section{Summary}

The current research has identified the need for enhancing the functional capabilities and efficiency of 3D surface defect detection and quantification. To date, this research has developed and successfully demonstrated;

(1) Generation of traceable and repeatable defect artefacts with different sizes.

(2) Measurement of surface feature using parallel optical coherence tomography instrument.

(3) Robust and automated measurement of defects using new MATLAB based algorithm, with high level of repeatability in quantification of defects in 3D.

(4) Traceable and mathematically well-defined defect soft-artefact to validate the algorithm.

(5) Measured industrial component using the implemented solution.

Further work is currently involved with refining algorithm capability and speed, developing point cloud stitching algorithm to cover larger area and exploring the applicability of this process to a broader group of real defects on a range of different operational surfaces.

\section{Acknowledgement}

The authors acknowledge support from the EPSRC Centre for Innovative Manufacturing in Intelligent Automation, in undertaking this research work under grant reference number $\mathrm{EP} / \mathrm{IO} 33467 / 1$.

\section{References}

[1] Lou S, Jiang X, Scott P, Application of the morphological alpha shape method to the extraction of topographical features from engineering surfaces, Measurement, 46(2), 1002-1008, 2013.

[2] Cubero, S, Aleixos, N, Moltó, E, Gómez-Sanchis, J, Blasco, J, Advances in machine vision applications for automatic inspection and quality evaluation of fruits and vegetables, Food and Bioprocess Technology, 4(4), 487-504, 2011

[3] Martínez, S, Ortega, J, García, J, García, A, A machine vision system for defect characterization on transparent parts with non-plane surfaces, Machine Vision and Applications, 23(1), 1-13, 2012 
[4] Lin H, Computer-aided visual inspection of surface defects in ceramic capacitors chips, J. Mater. Process Technol., 189(1), 19-25, 2007.

[5] Tsai D, Huang T, Automated surface inspection for statistical textures, Image Vision Comput., 21(4), 307-323, 2003.

[6] Tsai D, Lai S, Defect detection in periodically patterned surfaces using independent component analysis, Pattern Recognit., 41(9), 2812-2832, 2008

[7] Li W, Tsai D, Automatic saw-mark detection in multicrystalline solar wafer images, Solar Energy Mater. Solar Cells, 95(8), 2206-2220, 2011.

[8] ISO 16610 series. Geometrical Product Specifications (GPS) - Filtration. 2010.

[9] Lou S, Zeng W, Jiang X, Scott PJ. Robust filtration techniques in geometrical metrology and their comparison, International Journal of Auto. Comput., 10(1), 1-8, 2013.

[10] ISO 8785. Geometrical Product Specifications (GPS) - Surface imperfection. Terms, definitions and parameters, 1999.

[11] ISO 25178-2. Geometrical Product Specifications (GPS) - Surface texture: areal. Part 2. Terms, definitions and surface texture parameters, 2010.

[12] ISO 6508-1. Metallic material. Rockwell hardness test. Test method, 2006. 